\title{
The prevalence and mechanisms of metabolic syndrome in schizophrenia: a review
}

\author{
Evangelos Papanastasiou
}

\begin{abstract}
Metabolic syndrome (MetS), a constellation of central obesity, hypertension, dyslipidaemia and glucose intolerance, is highly prevalent in individuals with schizophrenia and conveys significant cardiovascular risk and mortality. Associated risk factors are female sex, some ethnic groups, advanced age, long duration of illness, smoking and exposure to antipsychotic agents. The prevalence of MetS varies across countries and psychiatric populations, and its development can be very rapid. Regular monitoring of all features of MetS is the cornerstone of its early detection and management. Future research needs to focus more on genetic determinants of MetS in the context of schizophrenic illness. This review aims to update the reader with the latest knowledge about the prevalence of MetS in schizophrenia and what might be the underlying pathophysiological mechanisms.
\end{abstract}

Keywords: antipsychotic-induced weight gain, cardiovascular risk factors, diabetes, dyslipidaemia, hypertension, incidence, metabolic syndrome, obesity, prevalence, schizophrenia

\section{Metabolic syndrome: introduction}

The metabolic syndrome (MetS, also known as syndrome $\mathrm{X}$, syndrome of chronic cardiovascular disease and Reaven's syndrome) is a constellation of different conditions, including abdominal obesity, insulin resistance, dyslipidaemia and elevated blood pressure. All components of the MetS (with obesity holding a central role in its development) have been recognized as independent risk factors for cardiovascular disease and so the presence of MetS is associated with other comorbidities such as the prothrombotic state, proinflammatory state, nonalcoholic fatty liver disease and reproductive disorders [Cornier et al. 2008]. Owing to its multiple components, many definitions have been given to MetS: by the World Health Organization (WHO) in 1985, by the European Group for the Study of Insulin Resistance in 1999, by the National Cholesterol Education Program's Adult Treatment Panel III (NCEPATP III) in 2001 (modified in 2003), by the American Association of Clinical Endocrinologists in 2003 and finally by the International Diabetes Federation (IDF) in 2006 (Table 1). In an attempt to unify criteria of MetS proposed by various organizations, a joint statement of the
IDF, the National Heart, Lung and Blood Institute, the American Heart Association, the World Heart Federation, the International Atherosclerosis Society and the International Association for the Study of Obesity suggested a revision of central obesity criteria to account for ethnic differences [Alberti et al. 2009]. According to the above, the diagnosis of MetS should be established if any three of the five criteria described below are present:

(1) Elevated waist circumference, according to population- and country-specific definitions (usually high thresholds for North America and North Europe, moderate thresholds for Asia, the Middle East, the Mediterranean, Africa, Central and South America and low thresholds for China and Japan).

(2) Elevated triglycerides $(\geq 150 \mathrm{mg} / \mathrm{dl})$ or drug treatment for dyslipidaemia.

(3) Reduced high-density lipoprotein (HDL) $(<40 \mathrm{mg} / \mathrm{dl})$ or drug treatment for hypercholesterolaemia.

(4) Elevated blood pressure (systolic $\geq 130$ $\mathrm{mmHg}$, diastolic $\geq 85 \mathrm{mmHg}$ ) or a history
Ther Adv Psychopharmacol (2013) 3(1) 33-51

DOI: $10.1177 /$ 2045125312464385

(C) The Author(s), 2012. Reprints and permissions: http://www.sagepub.co.uk/ journalsPermissions.nav
Correspondence to: Evangelos Papanastasiou, MD, MSc

CSI Lab, Department of Psychosis Studies, Institute of Psychiatry, KCL, De Crespigny Park, P063, Denmark Hill, Camberwell, London SE5 8AF, UK evangelos. papanastasiouRkcl.ac.uk 
Table 1. Criteria for the metabolic syndrome definition (adapted from Cornier et al. 2008, International Diabetes Federation, 2006).

\begin{tabular}{|c|c|c|c|c|}
\hline WHO (1998) & EGIR (1999) & $\begin{array}{l}\text { NCEP-ATP III (2001, } \\
\text { modified in 2003) }\end{array}$ & AACE (2003) & IDF (2006) \\
\hline $\begin{array}{l}(A) \text { and any two of } \\
(B),(C),(D) \text { or }(E)\end{array}$ & $\begin{array}{l}(A) \text { and any two of }(B) \text {, } \\
(C),(D),(E) \text { or }(F)\end{array}$ & \multirow[t]{2}{*}{$\begin{array}{l}\text { Three or more of }(A) \text {, } \\
(B),(C),(D) \text { or }(E)\end{array}$} & $\begin{array}{l}\text { (A) and any two of } \\
(B),(C) \text { or }(D)\end{array}$ & \multirow[t]{2}{*}{$\begin{array}{l}(A) \text { and any two of }(B) \text {, } \\
(C),(D) \text { or }(E)\end{array}$} \\
\hline $\begin{array}{l}\text { (A) High insulin } \\
\text { levels, impaired } \\
\text { glucose tolerance } \\
\text { or impaired fasting } \\
\text { glucose }\end{array}$ & $\begin{array}{l}\text { (A) Top } 25 \% \text { of the } \\
\text { fasting insulin values } \\
\text { among individuals } \\
\text { without diabetes }\end{array}$ & & \multirow[t]{3}{*}{$\begin{array}{l}\text { (A) Impaired glucose } \\
\text { tolerance }\end{array}$} & \\
\hline $\begin{array}{l}\text { (B) Abdominal } \\
\text { obesity: }\end{array}$ & $\begin{array}{l}\text { (B) Waist } \\
\text { circumference: }\end{array}$ & $\begin{array}{l}\text { (A) Waist } \\
\text { circumference: }\end{array}$ & & (A) Abdominal obesity: \\
\hline $\begin{array}{l}\text { waist-to-hip ratio > } \\
0.9, \mathrm{BMI} \geq 30 \mathrm{~kg} / \mathrm{m}^{2}, \\
\text { waist circumference } \\
>94 \mathrm{~cm}\end{array}$ & $\begin{array}{l}\geq 94 \mathrm{~cm} \text { for men, } \\
\geq 80 \mathrm{~cm} \text { for women }\end{array}$ & $\begin{array}{l}>102 \mathrm{~cm} \text { for men, } \\
>88 \mathrm{~cm} \text { for women }\end{array}$ & & $\begin{array}{l}\text { BMI } \geq 30 \mathrm{~kg} / \mathrm{m}^{2}, \text { waist } \\
\text { circumference } \geq 94 \mathrm{~cm} \\
\text { for men, } \geq 80 \mathrm{~cm} \text { for } \\
\text { women (of European } \\
\text { descent) }\end{array}$ \\
\hline $\begin{array}{l}\text { (C) Raised } \\
\text { triglycerides: }\end{array}$ & $\begin{array}{l}\text { (C) Raised } \\
\text { triglycerides: }\end{array}$ & $\begin{array}{l}\text { (B) Raised } \\
\text { triglycerides: }\end{array}$ & $\begin{array}{l}\text { (B) Raised } \\
\text { triglycerides: }\end{array}$ & $\begin{array}{l}\text { (B) Raised } \\
\text { triglycerides: }\end{array}$ \\
\hline$>150 \mathrm{mg} / \mathrm{dl}$ & $\geq 177 \mathrm{mg} / \mathrm{dl}$ & $\geq 150 \mathrm{mg} / \mathrm{dl}$ & $\geq 150 \mathrm{mg} / \mathrm{dl}$ & $\geq 150 \mathrm{mg} / \mathrm{dl}$ \\
\hline $\begin{array}{l}\text { (D) Reduced HDL } \\
\text { cholesterol: }\end{array}$ & $\begin{array}{l}\text { (D) Reduced HDL } \\
\text { cholesterol: }\end{array}$ & $\begin{array}{l}\text { (C) Reduced HDL } \\
\text { cholesterol: }\end{array}$ & $\begin{array}{l}\text { (C) Reduced HDL } \\
\text { cholesterol: }\end{array}$ & $\begin{array}{l}\text { (C) Reduced HDL } \\
\text { cholesterol: }\end{array}$ \\
\hline$<35 \mathrm{mg} / \mathrm{dl}$ & $<38.6 \mathrm{mg} / \mathrm{dl}$ & $\begin{array}{l}<40 \mathrm{mg} / \mathrm{dl} \text { for men, } \\
<50 \mathrm{mg} / \mathrm{dl} \text { for women }\end{array}$ & $\begin{array}{l}<40 \mathrm{mg} / \mathrm{dl} \text { for } \\
\text { men, }<50 \mathrm{mg} / \mathrm{dl} \text { for } \\
\text { women }\end{array}$ & $\begin{array}{l}<40 \mathrm{mg} / \mathrm{dl} \text { for men, }<50 \\
\mathrm{mg} / \mathrm{dl} \text { for women }\end{array}$ \\
\hline \multirow{4}{*}{$\begin{array}{l}\text { (E) Raised BP: } \\
>140 / 90 \mathrm{mmHg}\end{array}$} & (E) Raised BP: & (D) Raised BP: & \multirow{4}{*}{$\begin{array}{l}\text { (D) Raised BP: } \\
\geq 130 / 85 \mathrm{mmHg}\end{array}$} & (D) Raised BP: \\
\hline & $\begin{array}{l}\geq 140 / 90 \mathrm{mmHg} \text { or } \\
\text { antihypertensive } \\
\text { medication }\end{array}$ & $\geq 130 / 85 \mathrm{mmHg}$ & & $\begin{array}{l}\text { systolic } B P \geq 130 \mathrm{mmHg} \\
\text { or diastolic } B P \geq 85 \\
\mathrm{mmHg}\end{array}$ \\
\hline & $\begin{array}{l}\text { (F) Raised fasting } \\
\text { plasma glucose: }\end{array}$ & $\begin{array}{l}\text { (E) Raised fasting } \\
\text { plasma glucose: }\end{array}$ & & $\begin{array}{l}\text { (E) Raised fasting } \\
\text { plasma glucose: }\end{array}$ \\
\hline & $\geq 110 \mathrm{mg} / \mathrm{dl}$ & $\begin{array}{l}\geq 110 \mathrm{mg} / \mathrm{dl} ; \text { from } \\
2003 \mathrm{onwards} \\
\geq 100 \mathrm{mg} / \mathrm{dl}\end{array}$ & & $\begin{array}{l}\geq 100 \mathrm{mg} / \mathrm{dl} \text { or } \\
\text { previously diagnosed } \\
\text { type II diabetes }\end{array}$ \\
\hline
\end{tabular}

of hypertension or drug treatment for hypertension.

(5) Elevated fasting glucose $(\geq 100 \mathrm{mg} / \mathrm{dl})$ or drug treatment for hyperglycaemia.

The prevalence of MetS is increasing throughout the world but is partly dependent on the definition that is used to determine inclusion as well as the composition of the population being studied (i.e. sex, age, race and ethnicity) [Cornier et al. 2008]. Using the modified NCEP-ATP III criteria, the National Health and Nutrition Examination Survey (NHANES) compared data from a US cohort of 6423 adults (1988-1994) with a similar one of 6962 participants (19992006) and concluded that there has been an increase in age-adjusted MetS prevalence from $29.2 \%$ to $34.2 \%$ respectively over the years [Mozumdar and Liguori, 2011]. Efforts to control specific cardiovascular risk factors, such as blood pressure or total serum cholesterol levels, worldwide appear to have been partially effective since the 1980s (especially for countries with high income) [Danaei et al. 2011; Farzadfar et al. 2011]. Despite this, the mean body mass index (BMI) of the world's population has shown a constant increase in the last three decades, both 
in developed and developing countries [Finucane et al. 2011]. Although this increase in BMI is likely to be a positive thing in developing countries, as it indicates that more people have better nutrition, in developed countries this is giving rise to the so-called 'obesity epidemic'.

So it appears that MetS is common and increasing in the general population regardless of definition. Increased calorie intake and sedentary lifestyles have been implicated in the development of MetS worldwide, and without doubt constitutes a major public health risk. However, it is worth noting that certain population groups and more importantly certain patient groups have an even greater predisposition to developing MetS.

\section{Metabolic parameters of schizophrenia}

Schizophrenia is a highly heritable condition, which is associated with a dramatic reduction in lifespan; a meta-analysis of existing data has revealed a substantial gap between the health of people with schizophrenia and the general community [Saha et al. 2007]. Mortality in patients with schizophrenia is largely due to cardiovascular disease [Tandon et al. 2009]. Sudden cardiac death, often resulting from cardiac arrhythmias, is also an important cause of mortality [Koponen et al. 2008].

Schizophrenia has been associated with an increased risk of diabetes since the nineteenth century [Maudsley, 1979]. Henry Maudsley was one of the first psychiatrists to notice an association between diabetes and schizophrenia. This was prior to the development of antipsychotic treatments. Even today, a significant number of studies have demonstrated that antipsychotic naïve patients have impaired glucose tolerance, increased insulin resistance and increased visceral fat distribution compared with normal controls [Thakore et al. 2002; Venkatasubramanian et al. 2007; Fernandez-Egea et al. 2009]. More importantly, other studies have shown increased glucose intolerance in the siblings of people with schizophrenia and an increased prevalence of type II diabetes in the parents of subjects with nonaffective psychosis [Fernandez-Egea et al. 2008a, 2008b]. Recently, a Danish study found that having schizophrenia is associated with an at-risk allele for type II diabetes located in the TCF7L2 gene [Hansen et al. 2011]. These findings suggest that diabetes and schizophrenia may share familial risk factors or common genetic predisposition.
It has been estimated that in the USA as many as $60 \%$ of people with schizophrenia meet the criteria for MetS, as opposed to $30 \%$ for the general population [Mendelson, 2008]. Numerous studies have shown that overweight and diabetes are in general increased in people with schizophrenia, with a two- to fourfold increase in the risk of diabetes compared with the general population [Leucht et al. 2007a, 2007b]. This association, possibly predating the introduction of antipsychotic medication, has raised multiple hypotheses to account for the association between schizophrenia and diabetes by making reference to a number of intrinsic and extrinsic factors (Table 2).

\section{Rationale and objectives}

In this review we provide an update about MetS in schizophrenia. We aim to present data from original studies, which consider the MetS as an entity using any of the established definitions.

We set the following objectives at the beginning of our review:

(1) To provide a current estimate of the extent of MetS, with reference to its prevalence and incidence in populations of patients with schizophrenia.

(2) To identify factors that are associated with the development and presence of MetS; these could be either related to the environment (health habits, use of antipsychotic medication) or to characteristics of the individual patients (sex, age and duration of illness).

\section{Review methodology}

\section{Eligibility criteria}

Eligibility criteria were original articles published in English language until 2011. Studies were included on the basis of making reference to any established definition of MetS and excluded if they only described individual features of MetS. We included studies that adopted an adequately robust design, either cohorts, case-control, crosssectional or randomized controlled trials (RCTs).

\section{Information sources}

Information was obtained from the ISI Web of Knowledge platform (by Thomson Reuters), a 
Table 2. Hypotheses on the association between schizophrenia and diabetes ladapted from Leucht et al. [2007a, 2007b]).

\begin{tabular}{|c|c|c|c|}
\hline Environmental & Pharmacological & Biochemical & Other possible links \\
\hline $\begin{array}{l}\text { Sedentary lifestyle, lack of } \\
\text { exercise }\end{array}$ & $\begin{array}{l}\text { Weight-inducing and } \\
\text { diabetogenic effect of } \\
\text { some antipsychotic } \\
\text { drugs }\end{array}$ & $\begin{array}{l}\text { Overexcitement of the } \\
\text { sympathetic system, } \\
\text { hypersecretion of adrenalin }\end{array}$ & Inefficiency of the liver \\
\hline Smoking, substance abuse & $\begin{array}{l}\text { Effect of antipsychotic } \\
\text { medication on liver } \\
\text { metabolism }\end{array}$ & $\begin{array}{l}\text { Dysregulation of the } \\
\text { hypothalamic-pituitary- } \\
\text { adrenal axis }\end{array}$ & $\begin{array}{l}\text { Positive family history } \\
\text { of diabetes (genetic) }\end{array}$ \\
\hline $\begin{array}{l}\text { Adverse diet, poor nutrition, } \\
\text { high intake of fast food, lack } \\
\text { of understanding of dietary } \\
\text { principles }\end{array}$ & & $\begin{array}{l}\text { Hyper-cortisolaemia, } \\
\text { excessive visceral } \\
\text { fat, hyperglycaemia, } \\
\text { hyperinsulinaemia, insulin } \\
\text { resistance }\end{array}$ & Low birth weight \\
\hline Lethargy, sedation & & Anti-insulin factor & \\
\hline $\begin{array}{l}\text { Inadequate medical or public } \\
\text { healthcare, poor adherence } \\
\text { to prescribed medication }\end{array}$ & & $\begin{array}{l}\text { Abnormal glucose } \\
\text { metabolism }\end{array}$ & \\
\hline $\begin{array}{l}\text { Stressful experiences } \\
\text { (hospitalization, psychotic } \\
\text { symptoms) }\end{array}$ & & $\begin{array}{l}\text { Sex hormones and } \\
\text { oestradiol mediating weight } \\
\text { gain }\end{array}$ & \\
\hline
\end{tabular}

comprehensive database that incorporates the Web of Science (1970 to present) and MEDLINE (1950 to present) and also includes articles from PsychINFO and the Cochrane Review Database.

\section{Search}

We searched articles using the terms: Title $=$ (schizophrenia) AND Title = (metabolic syndrome), published until year 2011. Our initial search generated 119 hits. We completed our search by checking against previously published reviews and extracting additional articles (Table 3).

\section{Study selection}

Screening of articles was based on titles and abstract reading. Only articles fulfilling our eligibility criteria were included, and full texts were subsequently obtained. Special care was taken to avoid duplications and group together different articles describing the same study population.

\section{Outcome}

A total of 47 original studies were identified (Table 4).

Several researchers have tried to summarize the current evidence on MetS in schizophrenia in numerous systematic or selective reviews. Most reviews focus on studies of epidemiological interest but also attempt to address the pathophysiological connections between MetS and schizophrenia. A group of reviews focus particularly on studies of metabolic features associated with the use of second-generation antipsychotics. A significant number of reviews that focus on behavioural and pharmacological interventions targeting metabolic disturbances in schizophrenia and severe mental illness are also available, but they are not mentioned here as they are beyond the scope of this article.

\section{Description/discussion of studies}

Observational studies on prevalence and incidence of MetS were conducted in several countries, and the most numerous publications come from the USA, followed by Belgium and Finland. The great majority of studies employed a crosssectional design, and fewer were case-control and cohort studies. Only two RCTs were included, the first comparing the incidence of MetS among patients treated with aripiprazole versus olanzapine and placebo [L'Italien et al. 2007] and the second comparing prevalence rates of MetS developed following 6 weeks of treatment of young unmedicated drug-naïve patients with haloperidol, risperidone or olanzapine [Saddichha et al. 2008]. The composition 
Table 3. Published descriptive reviews of metabolic syndrome (MetS) in schizophrenia.

\begin{tabular}{|c|c|c|c|}
\hline Authors, year & Type of review & No. of articles reviewed & Area of main focus \\
\hline $\begin{array}{l}\text { Meyer et al. } \\
\text { [2005a] }\end{array}$ & Selective & & $\begin{array}{l}\text { Prevalence of MetS, } \\
\text { pathophysiology }\end{array}$ \\
\hline $\begin{array}{l}\text { Leucht et al. } \\
\text { [2007a, 2007b] }\end{array}$ & Systematic & 40 & $\begin{array}{l}\text { Prevalence of MetS, } \\
\text { pathophysiology, role of } \\
\text { antipsychotics }\end{array}$ \\
\hline $\begin{array}{l}\text { Lindley et al. } \\
\text { [2008] }\end{array}$ & Selective & & $\begin{array}{l}\text { Insulin resistance, } \\
\text { pathophysiology, lifestyle } \\
\text { features }\end{array}$ \\
\hline Mendelson [2008] & Selective & & $\begin{array}{l}\text { Pathophysiology, N-methyl-D- } \\
\text { aspartate receptor }\end{array}$ \\
\hline $\begin{array}{l}\text { De Hert et al. } \\
\text { [2009] }\end{array}$ & Systematic & 36 & Prevalence and incidence of MetS \\
\hline $\begin{array}{l}\text { Meyer and Stahl } \\
\text { [2009] }\end{array}$ & Systematic & 11 & $\begin{array}{l}\text { Prevalence of MetS, role of } \\
\text { triglycerides }\end{array}$ \\
\hline $\begin{array}{l}\text { Mitchell et al. } \\
\text { [2011] }\end{array}$ & $\begin{array}{l}\text { Systematic, } \\
\text { meta-analysis }\end{array}$ & 77 & $\begin{array}{l}\text { Prevalence and predictors of } \\
\text { MetS accounting for subgroup } \\
\text { differences }\end{array}$ \\
\hline $\begin{array}{l}\text { Mitchell et al. } \\
\text { [2012b] }\end{array}$ & $\begin{array}{l}\text { Systematic, } \\
\text { meta-analysis }\end{array}$ & 123 & $\begin{array}{l}\text { Prevalence of MetS in first } \\
\text { episode schizophrenia and in } \\
\text { unmedicated patients compared } \\
\text { with patients established on } \\
\text { medication }\end{array}$ \\
\hline $\begin{array}{l}\text { Taylor and } \\
\text { McAskill [2000] }\end{array}$ & Systematic & 74 & $\begin{array}{l}\text { Atypical antipsychotics and } \\
\text { weight gain }\end{array}$ \\
\hline Jin et al. [2004] & Systematic & 27 & $\begin{array}{l}\text { Atypical antipsychotics and } \\
\text { glucose dysregulation }\end{array}$ \\
\hline $\begin{array}{l}\text { Meyer and Koro } \\
\text { [2004] }\end{array}$ & Systematic & 48 & Antipsychotics and serum lipids \\
\hline Newcomer [2005] & Comprehensive & $>200$ & $\begin{array}{l}\text { Atypical antipsychotics and } \\
\text { glucose dysregulation, lipids } \\
\text { metabolism }\end{array}$ \\
\hline Smith et al. [2008] & $\begin{array}{l}\text { Systematic, } \\
\text { meta-analysis }\end{array}$ & 14 & $\begin{array}{l}\text { Antipsychotics and risk for } \\
\text { diabetes in schizophrenia }\end{array}$ \\
\hline Simon et al. [2009] & Systematic & 45 & $\begin{array}{l}\text { Atypical antipsychotics and dose- } \\
\text { dependent metabolic features }\end{array}$ \\
\hline $\begin{array}{l}\text { De Hert et al. } \\
\text { [2012a] }\end{array}$ & Systematic & $>100$ & $\begin{array}{l}\text { Metabolic and cardiovascular } \\
\text { risks of various antipsychotic } \\
\text { medication in adults and } \\
\text { adolescents }\end{array}$ \\
\hline $\begin{array}{l}\text { De Hert et al. } \\
\text { [2012b] }\end{array}$ & $\begin{array}{l}\text { Systematic, } \\
\text { meta-analysis }\end{array}$ & 56 & $\begin{array}{l}\text { Weight gain and metabolic } \\
\text { adverse events of asenapine, } \\
\text { iloperidone, lurasidone and } \\
\text { paliperidone }\end{array}$ \\
\hline
\end{tabular}

of the study group varied, however male patients appeared to be over represented. In most studies the mean age of participants in the study group was over 40 years of age, and only a few studies referred to patients with first episode schizophrenia [Attux et al. 2007; Saddichha et al. 2007; De Hert et al. 2008b; Saddichha et al. 2008; Curtis et al. 2011]. The predominant diagnosis of patients studied was schizophrenia, however a great number of studies also included patients with schizoaffective disorder and other psychotic disorders. In almost all studies, patients were medicated with first- and second-generation antipsychotic drugs (FGAs, SGAs) and only one cross-sectional and two case-control studies referred to drug-naïve patients [Saddichha et al. 
Table 4. Original observational studies on metabolic syndrome and schizophrenia.

\begin{tabular}{|c|c|c|c|c|c|c|c|}
\hline Authors, year & Country & Design & $\begin{array}{l}\text { Study } \\
\text { group }(N)\end{array}$ & Diagnoses & $\begin{array}{l}\text { Medication } \\
\text { status }\end{array}$ & $\begin{array}{l}\text { Definition criteria } \\
\text { for MetS }\end{array}$ & $\begin{array}{l}\text { Total prevalence } \\
\text { of MetS }(\%)\end{array}$ \\
\hline $\begin{array}{l}\text { Heiskanen et al. } \\
\text { [2003] }\end{array}$ & Finland & $\begin{array}{l}\text { Cross } \\
\text { sectional }\end{array}$ & 35 & SCZ, SCZA & FGA, SGA & NCEP-ATP III & 37.0 \\
\hline $\begin{array}{l}\text { Almeras et al. } \\
\text { [2004] }\end{array}$ & Canada & $\begin{array}{l}\text { Cross } \\
\text { sectional }\end{array}$ & 87 & SCZ, PSYCH & SGA & NCEP-ATP III & Not calculated \\
\hline $\begin{array}{l}\text { Basu et al. } \\
{[2004]}\end{array}$ & USA & $\begin{array}{l}\text { Cross } \\
\text { sectional }\end{array}$ & 33 & SCZA & FGA, SGA, MS & NCEP-ATP III & 42.4 \\
\hline $\begin{array}{l}\text { Cohn et al. } \\
\text { [2004] }\end{array}$ & Canada & $\begin{array}{l}\text { Case } \\
\text { controls }\end{array}$ & 240 & SCZ, SCZA & FGA, SGA & NCEP-ATP III & Not calculated \\
\hline $\begin{array}{l}\text { Kato et al. } \\
{[2004]}\end{array}$ & USA & $\begin{array}{l}\text { Cross } \\
\text { sectional }\end{array}$ & 48 & $\mathrm{SCZ}$ & FGA, SGA & NCEP-ATP III & 63.0 \\
\hline $\begin{array}{l}\text { McEvoy et al. } \\
\text { [2005] }\end{array}$ & USA & $\begin{array}{l}\text { Cross } \\
\text { sectional }\end{array}$ & 689 & $\mathrm{SCZ}$ & FGA, SGA & $\begin{array}{l}\text { NCEP-ATP III, } \\
\text { NCEP-ATP III mod }\end{array}$ & $40.9 ; 42.7$ \\
\hline $\begin{array}{l}\text { Meyer et al. } \\
\text { [2005b] }\end{array}$ & USA & $\begin{array}{l}\text { Cross } \\
\text { sectional }\end{array}$ & 1231 & $\mathrm{SCZ}$ & FGA, SGA & NCEP-ATP III & 35.8 \\
\hline $\begin{array}{l}\text { Saari et al. } \\
\text { [2005] }\end{array}$ & Finland & $\begin{array}{l}\text { Case } \\
\text { controls }\end{array}$ & 158 & SCZ, PSYCH & FGA, SGA & NCEP-ATP III & 19.4 \\
\hline $\begin{array}{l}\text { Straker et al. } \\
\text { [2005] }\end{array}$ & USA & $\begin{array}{l}\text { Cross } \\
\text { sectional }\end{array}$ & 89 & $\mathrm{SCZ}, \mathrm{MD}$ & FGA, SGA & NCEP-ATP III & 28.0 \\
\hline $\begin{array}{l}\text { Correll et al. } \\
\text { [2006] }\end{array}$ & USA & $\begin{array}{l}\text { Cross } \\
\text { sectional }\end{array}$ & 367 & $\begin{array}{l}\text { SCZ, SCZA, } \\
\text { MD }\end{array}$ & SGA, MS & NCEP-ATP III, IDF & 42.0 \\
\hline $\begin{array}{l}\text { De Hert et al. } \\
\text { [2006b] }\end{array}$ & Belgium & $\begin{array}{l}\text { Cross } \\
\text { sectional }\end{array}$ & 430 & SCZ, SCZA & FGA, SGA & $\begin{array}{l}\text { NCEP-ATP III, } \\
\text { NCEP-ATP III mod, } \\
\text { IDF }\end{array}$ & $28.4 ; 32.3 ; 36.0$ \\
\hline $\begin{array}{l}\text { Hagg et al. } \\
\text { [2006] }\end{array}$ & Sweden & $\begin{array}{l}\text { Cross } \\
\text { sectional }\end{array}$ & 269 & $\mathrm{SCZ}$ & FGA, SGA & NCEP-ATP III & 34.6 \\
\hline $\begin{array}{l}\text { Lamberti et al. } \\
\text { [2006] }\end{array}$ & USA & $\begin{array}{l}\text { Case } \\
\text { controls }\end{array}$ & 93 & $\begin{array}{l}\text { SCZ and } \\
\text { others }\end{array}$ & CLOZ & NCEP-ATP III & 53.8 \\
\hline $\begin{array}{l}\text { Meyer et al. } \\
{[2006]}\end{array}$ & USA & $\begin{array}{l}\text { Cross } \\
\text { sectional }\end{array}$ & 80 & SCZ, SCZA & Not stated & $\begin{array}{l}\text { NCEP-ATP III, } \\
\text { NCEP-ATP III mod }\end{array}$ & $48.8 ; 56.3$ \\
\hline $\begin{array}{l}\text { Attux et al. } \\
\text { [2007] }\end{array}$ & Brazil & $\begin{array}{l}\text { Cross } \\
\text { sectional }\end{array}$ & 44 & $\mathrm{SCZ}$ & FGA, SGA & NCEP-ATP III & Not calculated \\
\hline $\begin{array}{l}\text { Correll et al. } \\
\text { [2007] }\end{array}$ & USA & $\begin{array}{l}\text { Cross } \\
\text { sectional }\end{array}$ & 364 & SCZ, MD & FGA, SGA & NCEP-ATP III & 37.4 \\
\hline $\begin{array}{l}\text { De Hert et al. } \\
\text { [2007] }\end{array}$ & Belgium & $\begin{array}{l}\text { Cross } \\
\text { sectional }\end{array}$ & 294 & $\mathrm{SCZ}$ & SGA & NCEP-ATP III, IDF & $28.2 ; 35.7$ \\
\hline $\begin{array}{l}\text { L'Italien et al. } \\
\text { [2007] }\end{array}$ & USA & $\mathrm{RCT}$ & 1175 & $\mathrm{SCZ}$ & Not stated & NCEP-ATP III & Not calculated \\
\hline $\begin{array}{l}\text { Mackin et al. } \\
\text { [2007] }\end{array}$ & UK & $\begin{array}{l}\text { Case } \\
\text { controls }\end{array}$ & 90 & SCZ, MISC & FGA, SGA, MS & IDF & 33.3 \\
\hline $\begin{array}{l}\text { Saddichha et al. } \\
\text { [2007] }\end{array}$ & India & $\begin{array}{l}\text { Cross } \\
\text { sectional }\end{array}$ & 30 & $\mathrm{SCZ}$ & $\begin{array}{l}\text { Drug naive, } \\
\text { FGA/SGA }\end{array}$ & $\begin{array}{l}\text { NCEP-ATP III mod, } \\
\text { IDF }\end{array}$ & Not calculated \\
\hline $\begin{array}{l}\text { Srisurapanont } \\
\text { et al. [2007] }\end{array}$ & Thailand & $\begin{array}{l}\text { Cross } \\
\text { sectional }\end{array}$ & 57 & $\mathrm{SCZ}$ & FGA, SGA & $\begin{array}{l}\text { NCEP-ATP III, } \\
\text { NCEP-ATP III mod, } \\
\text { IDF }\end{array}$ & 22.8 \\
\hline $\begin{array}{l}\text { Suvisaari et al. } \\
\text { [2007] }\end{array}$ & Finland & $\begin{array}{l}\text { Case } \\
\text { controls }\end{array}$ & 118 & SCZ, PSYCH & FGA, SGA & NCEP-ATP III & 36.2 \\
\hline $\begin{array}{l}\text { Teixeira and } \\
\text { Rochal [2007] }\end{array}$ & Brazil & $\begin{array}{l}\text { Cross } \\
\text { sectional }\end{array}$ & 170 & $\begin{array}{l}\text { SCZ, SCZA, } \\
\text { MD }\end{array}$ & Not stated & NCEP-ATP III mod & 31.8 \\
\hline $\begin{array}{l}\text { Tirupati and } \\
\text { Chua [2007] }\end{array}$ & New Zealand & $\begin{array}{l}\text { Cross } \\
\text { sectional }\end{array}$ & 221 & $\begin{array}{l}\text { SCZ, SCZA, } \\
\text { MD }\end{array}$ & FGA, SGA, MS & IDF & 68.0 \\
\hline $\begin{array}{l}\text { Boke et al. } \\
\text { [2008] }\end{array}$ & Turkey & $\begin{array}{l}\text { Cross } \\
\text { sectional }\end{array}$ & 231 & $\mathrm{SCZ}$ & Not stated & IDF & 32.0 \\
\hline $\begin{array}{l}\text { Cerit et al. } \\
\text { [2008] }\end{array}$ & Turkey & $\begin{array}{l}\text { Cross } \\
\text { sectional }\end{array}$ & 100 & $\mathrm{SCZ}$ & FGA, SGA & $\begin{array}{l}\text { NCEP-ATP III, } \\
\text { NCEP-ATP III mod, } \\
\text { IDF }\end{array}$ & $21.0 ; 34.0 ; 41.0$ \\
\hline
\end{tabular}


Table 4. (Continued)

\begin{tabular}{|c|c|c|c|c|c|c|c|}
\hline Authors, year & Country & Design & $\begin{array}{l}\text { Study } \\
\text { group }(N)\end{array}$ & Diagnoses & $\begin{array}{l}\text { Medication } \\
\text { status }\end{array}$ & $\begin{array}{l}\text { Definition criteria } \\
\text { for MetS }\end{array}$ & $\begin{array}{l}\text { Total prevalence } \\
\text { of MetS }(\%)\end{array}$ \\
\hline $\begin{array}{l}\text { Correll et al. } \\
\text { [2008] }\end{array}$ & USA & $\begin{array}{l}\text { Cross } \\
\text { sectional }\end{array}$ & 185 & $\mathrm{SCZ}, \mathrm{MD}$ & FGA, SGA, MS & $\begin{array}{l}\text { NCEP-ATP III, } \\
\text { NCEP-ATP III mod }\end{array}$ & $45.9 ; 54.0$ \\
\hline $\begin{array}{l}\text { Meyer et al. } \\
\text { [2008] }\end{array}$ & USA & $\begin{array}{l}\text { Cross } \\
\text { sectional }\end{array}$ & 660 & SCZ & Not stated & NCEP-ATP III mod & Not calculated \\
\hline $\begin{array}{l}\text { Saddichha et al. } \\
\text { [2008] }\end{array}$ & India & $\mathrm{RCT}$ & 99 & SCZ & $\begin{array}{l}\text { Drug naive, } \\
\text { FGA/SGA }\end{array}$ & $\begin{array}{l}\text { NCEP-ATP III mod, } \\
\text { IDF }\end{array}$ & $10.1 ; 18.2$ \\
\hline $\begin{array}{l}\text { Sicras-Mainar } \\
\text { et al. [2008] }\end{array}$ & Spain & $\begin{array}{l}\text { Case } \\
\text { controls }\end{array}$ & 742 & SCZ & FGA, SGA & $\begin{array}{l}\text { NCEP-ATP III mod, } \\
\text { IDF }\end{array}$ & 26.6 \\
\hline $\begin{array}{l}\text { Van Winkel } \\
\text { et al. [2008] }\end{array}$ & Belgium & Cohort & 503 & SCZ & FGA, SGA, MS & NCEP-ATP III mod & 28.8 \\
\hline $\begin{array}{l}\text { Huang et al. } \\
\text { [2009] }\end{array}$ & Taiwan & $\begin{array}{l}\text { Cross } \\
\text { sectional }\end{array}$ & 650 & SCZ, SCZA & FGA, SGA & NCEP-ATP III mod & 34.9 \\
\hline Lee et al. [2009] & Korea & $\begin{array}{l}\text { Cross } \\
\text { sectional }\end{array}$ & 271 & SCZ & Not stated & NCEP-ATP III mod & 33.6 \\
\hline $\begin{array}{l}\text { Rezaei et al. } \\
\text { [2009] }\end{array}$ & Iran & $\begin{array}{l}\text { Cross } \\
\text { sectional }\end{array}$ & 372 & $\mathrm{SCZ}$ & FGA, SGA & $\begin{array}{l}\text { NCEP-ATP III, } \\
\text { NCEP-ATP III mod, } \\
\text { IDF }\end{array}$ & $27.4 ; 37.6 ; 38.7$ \\
\hline $\begin{array}{l}\text { Schorr et al. } \\
\text { [2009] }\end{array}$ & Netherlands & $\begin{array}{l}\text { Cross } \\
\text { sectional }\end{array}$ & 433 & $\begin{array}{l}\text { SCZ, SCZA, } \\
\text { PSYCH }\end{array}$ & SGA & NCEP-ATP III & 35.6 \\
\hline Kim et al. [2010] & Korea & $\begin{array}{l}\text { Cross } \\
\text { sectional }\end{array}$ & 184 & SCZ & Not stated & NCEP-ATP III & 29.8 \\
\hline $\begin{array}{l}\text { Koponen et al. } \\
\text { [2010] }\end{array}$ & Finland & $\begin{array}{l}\text { Cross } \\
\text { sectional }\end{array}$ & 31 & SCZ & FGA, SGA & NCEP-ATP III, IDF & 29.0 \\
\hline $\begin{array}{l}\text { Kraemer et al. } \\
{[2010,2011]}\end{array}$ & Germany & $\begin{array}{l}\text { Cross } \\
\text { sectional, } \\
\text { longitudinal }\end{array}$ & 476 & SCZ & FGA, SGA & NCEP-ATP III & $\begin{array}{l}44.3 \text { (baseline) } \\
49.6 \text { ( } 3 \\
\text { months post } \\
\text { antipsychotic) }\end{array}$ \\
\hline $\begin{array}{l}\text { Padmavati et al. } \\
\text { [2010] }\end{array}$ & India & $\begin{array}{l}\text { Case } \\
\text { controls }\end{array}$ & 51 & SCZ & Drug naive & IDF & 3.9 \\
\hline $\begin{array}{l}\text { Curtis et al. } \\
\text { [2011] }\end{array}$ & Australia & $\begin{array}{l}\text { Cross } \\
\text { sectional }\end{array}$ & 64 & PSYCH & Not stated & IDF & 12.5 \\
\hline $\begin{array}{l}\text { Kang et al. } \\
\text { [2011] }\end{array}$ & Korea & $\begin{array}{l}\text { Cross } \\
\text { sectional }\end{array}$ & 957 & SCZ, SCZA & Not stated & NCEP-ATP III mod & 43.4 \\
\hline Lee et al. [2011] & Korea & $\begin{array}{l}\text { Cross } \\
\text { sectional }\end{array}$ & 145 & SCZ & SGA & NCEP-ATP III & 31.7 \\
\hline $\begin{array}{l}\text { Subashini et al. } \\
\text { [2011] }\end{array}$ & India & $\begin{array}{l}\text { Cross } \\
\text { sectional }\end{array}$ & 153 & SCZ & FGA, SGA & IDF & 34.4 \\
\hline $\begin{array}{l}\text { Yazici et al. } \\
\text { [2011] }\end{array}$ & Turkey & $\begin{array}{l}\text { Cross } \\
\text { sectional }\end{array}$ & 319 & SCZ & FGA, SGA & $\begin{array}{l}\text { NCEP-ATP III, } \\
\text { NCEP-ATP III mod, } \\
\text { IDF }\end{array}$ & $34.2 ; 37.0 ; 41.7$ \\
\hline $\begin{array}{l}\text { Bobes et al. } \\
\text { [2007]; Rejas } \\
\text { et al. [2008] }\end{array}$ & Spain & $\begin{array}{l}\text { Cross } \\
\text { sectional }\end{array}$ & 1452 & SCZ, SCZA & FGA, SGA & $\begin{array}{l}\text { NCEP-ATP III, } \\
\text { NCEP-ATP III mod }\end{array}$ & $24.6 ; 25.5$ \\
\hline $\begin{array}{l}\text { De Hert et al. } \\
{[2008 a, 2010]}\end{array}$ & Europe & $\begin{array}{l}\text { Cross } \\
\text { sectional }\end{array}$ & 2270 & SCZ & FGA, SGA & NCEP-ATP III mod & 33.9 \\
\hline $\begin{array}{l}\text { Sugawara et al. } \\
\text { [2011] }\end{array}$ & Japan & $\begin{array}{l}\text { Case } \\
\text { controls }\end{array}$ & 1186 & SCZ, SCZA & Not stated & $\begin{array}{l}\text { NCEP-ATP III mod, } \\
\text { IDF }\end{array}$ & $\begin{array}{l}\text { Total: } \\
27.5 ; 25.4 \\
\text { Inpatients: } \\
15.8 ; 14.7 \\
\text { Outpatients } \\
48.1 ; 44.1\end{array}$ \\
\hline
\end{tabular}

FGA, first-generation antipsychotics (conventional or typical); IDF, International Diabetes Federation; MD, affective disorders (including bipolar disorder): MS, mood stabilizers; NCEP-ATP III, National Cholesterol Education Programme - Adult Treatment Panel III; NCEP-ATP III mod, modified National Cholesterol Education Programme criteria, fasting glucose reduced from over $110 \mathrm{mg} / \mathrm{dl}$ to over $100 \mathrm{mg} / \mathrm{dl}$; PSYCH, other psychotic disorders; RCT, randomized controlled trial; SCZ, schizophrenia; SCZA, schizoaffective disorder, SGA, second-generation antipsychotics (atypical). 
2007, 2008; Padmavati et al. 2010]. Most studies used the NCEP-ATP III definition while some studies also calculated MetS rates by using the IDF definition.

\section{Overall prevalence rates}

Prevalence rates varied largely across studies. This possibly reflected the epidemiological versatility of the groups of patients studied, and factors such as age, sex, ethnicity, medication status, smoking, duration of illness and country of origin affected the final outcome. The lowest prevalence rate reported was $3.9 \%$, originating from an Indian population of 51 unmedicated, drug-naïve young outpatients (mean age 26.9) with chronic schizophrenia, and was based on the IDF definition of MetS [Padmavati et al. 2010]. The highest prevalence rate reported, $68 \%$, derived from a study of 221 psychotic inpatients and outpatients (mean age 37.9) from a New Zealand rehabilitation setting, who were treated with a combination of FGAs and SGAs (authors used the IDF criteria) [Tirupati and Chua, 2007]. Those two studies clearly showed how MetS rates vary between two completely different populations of patients with psychosis, who can be placed at the extremes of a spectrum in terms of their epidemiological features and medication status.

When different criteria were used to calculate MetS in the same population, outcome rates also varied, with IDF criteria usually generating the highest rates and NCEP-ATP III modified criteria the lowest [McEvoy et al. 2005; Correll et al. 2006, 2008; De Hert et al. 2006a and 2006b, 2007; Meyer et al. 2006; Bobes et al. 2007; Cerit et al. 2008; Rejas et al. 2008; Saddichha et al. 2008; Rezaei et al. 2009; Sugawara et al. 2010; Yazici et al. 2011]. Those studies that also included a control group revealed that the rates of MetS in patients with schizophrenia were at least twice as high compared with the general population [Cohn et al. 2004; McEvoy et al. 2005; Saari et al. 2005; Lamberti et al. 2006; Mackin et al. 2007; Sugawara et al. 2010]. This effect was usually more prominent in younger age groups and tended to be attenuated or even reversed in older age groups [McEvoy et al. 2005; Lamberti et al. 2006; Sugawara et al. 2010; Yazici et al. 2011]. Only one study from India reported a higher prevalence rate in the general population compared with patients with schizophrenia [Padmavati et al. 2010].

\section{Metabolic syndrome and sex}

Numerous studies compared prevalence rates between men and women. Most studies revealed substantially increased prevalence rates of MetS in women [Cohn et al. 2004; Kato et al. 2004; McEvoy et al. 2005; Correll et al. 2006; De Hert et al. 2006b; Hagg et al. 2006; Lamberti et al. 2006; Meyer et al. 2006; Bobes et al. 2007; Teixeira and Rochal, 2007; Cerit et al. 2008; Rejas et al. 2008; Sicras-Mainar et al. 2008; Huang et al. 2009; Rezaei et al. 2009; Yazici et al. 2011], up to threefold compared with men [Rezaei et al. 2009]. Fewer studies reported a slight predominance of male sex in MetS rates or no significant differences in rates of MetS across sexes [Heiskanen et al. 2003; Basu et al. 2004; Tirupati and Chua, 2007; Correll et al. 2008; Koponen et al. 2010; Kraemer et al. 2010; Sugawara et al. 2010, 2011].

\section{Metabolic syndrome and age}

The prevalence of MetS increases with age in the general population and a similar trend was generally observed in patients with schizophrenia. Quite a few studies have included cluster analysis of age groups, and an association between rates of MetS and age of participants was usually demonstrated [Cohn et al. 2004; McEvoy et al. 2005; Hagg et al. 2006; Lamberti et al. 2006; Bobes et al. 2007; Suvisaari et al. 2007; Cerit et al. 2008; Rejas et al. 2008; Huang et al. 2009; Sugawara et al. 2010, 2011; Kang et al. 2011; Yazici et al. 2011]. The pattern of this association was not always clear and depends on the level of age clusters studied by researchers. Some studies though revealed a peak of MetS rates in the third, fourth or fifth decade of life with subsequent decline in MetS rates in later life [Hagg et al. 2006; Sugawara et al. 2010]. This effect was not consistent through all studies, and it was not present in the same way in both sexes. A single study showed that women with schizophrenia tended to reach a peak in MetS rates in the forth decade of life, while men reach a peak in the third decade [Sugawara et al. 2010]. This finding could also reflect the fact that men tend to have an earlier onset of schizophrenia than women and so are exposed longer to this condition. It is of interest though that when agecluster analysis was applied to the same population, considering the type of psychiatric setting, more complex patterns were revealed. Both male and female inpatients showed a peak in MetS rates in their fourth decade of life with a further decline, while outpatients presented an atypical 
pattern of MetS prevalence changes, with men scoring higher in their third, fifth and seventh decades and women in their fourth and seventh decades [Sugawara et al. 2011]. However, one should be very cautious in trying to interpret the kind of data that stem from a single study.

\section{Metabolic syndrome and medication}

Only three studies included antipsychotic-naïve patients and reported rates of MetS lower than the rates described in the majority of studies. It is of note that two of these studies referred to drugnaïve patients who had been medicated for only 6 weeks with antipsychotic agents, and they developed MetS in response to this [Saddichha et al. 2007, 2008], and the third study referred to a very rare population of drug-naïve, unmedicated patients with an extremely low prevalence of MetS, as discussed above [Padmavati et al. 2010]. A German study explored the prevalence of MetS in patients with treated or untreated schizophrenia at baseline and at 3 months after initiation or switch of antipsychotic treatment. The authors reported an increase from $44.3 \%$ to $49.6 \%$, and also described the lowest baseline MetS prevalence $(24.7 \%)$ in previously unmedicated patients [Kraemer et al. 2011]. The lack of further studies on drug-naïve patients is an anticipated source of bias in any effort to explore the role of antipsychotic medication in the development of MetS. However, numerous studies attempted to compare various antipsychotics or groups of antipsychotics (FGAs versus SGAs) in terms of their contribution to MetS [Almeras et al. 2004; Kato et al. 2004; Straker et al. 2005; Correll et al. 2006, 2007; Hagg et al. 2006; L'Italien et al. 2007; Suvisaari et al. 2007; Tirupati et al. 2007; Cerit et al. 2008; De Hert et al. 2008b; Meyer et al. 2008; Saddichha et al. 2008; Huang et al. 2009; Rezaei et al. 2009; Schorr et al. 2009; Lee et al. 2011]. Consistent findings across these studies found that MetS was more likely with SGAs over FGAs, polypharmacy over monopharmacy and high-potency over low-potency agents. For individual antipsychotics, clozapine and olanzapine appeared to be related to higher MetS rates than other antipsychotic agents.

\section{Metabolic syndrome and ethnicity}

Very few studies attempted to address the issue of ethnicity when MetS rates are calculated [Basu et al. 2004; Kato et al. 2004; McEvoy et al. 2005;
Straker et al. 2005; Correll et al. 2006; Lamberti et al. 2006]. Black African and Hispanic patients appeared to present with higher rates of MetS, however some studies found rates to be similar to white populations. However, outcomes appeared to be quite inconsistent. It is of note that despite Indian and Asian populations having a predisposition to develop diabetes, studies of patients with schizophrenia originating from these populations usually reported lower prevalence rates of MetS compared with white and black patients. Of course this observation can also reflect the lower rates of prescribing atypical antipsychotics in developing versus developed countries.

\section{Metabolic syndrome and duration of pscyhotic illness or type of psychiatric setting}

Only three studies were identified which looked at duration of psychotic illness in the calculation of MetS rates. One prospective cross-sectional study calculated MetS prevalence across four clusters of illness duration and demonstrated a clear association: the longer the participants had had psychosis, the higher the rates of MetS [De Hert et al. 2006a]. Two more studies (referring to the same population) failed to reveal an association between chronicity of illness and higher prevalence of MetS [Bobes et al. 2007; Rejas et al. 2008]. A recent study from Japan revealed substantial differences in rates of MeS between inpatients and outpatients with schizophrenia, with outpatients presenting with almost threefold the prevalence of MetS compared with inpatients ( $48.1 \%$ versus $15.8 \%)$. This remarkable difference possibly reflects the fact that schizophrenia inpatients in Japan typically have long hospital stays, during which they receive controlled diets and occupational therapy [Sugawara et al. 2011].

\section{Metabolic syndrome and lifestyle habits}

Though some studies described lifestyle characteristics of their population, only a few chose to describe how these reflected MetS rates. Only four studies distinguished between smokers and nonsmokers when MetS rates were calculated [Lamberti et al. 2006; Cerit et al. 2008; Rezaei et al. 2009; Schorr et al. 2009]. In three of these, smokers appeared to have higher rates of MetS compared with either nonsmokers or the whole study population, while only one study showed similar rates between the two groups [Cerit et al. 2008]. 
Incidence of metabolic syndrome

A minority of studies calculated incidence rates of MetS [Attux et al. 2007; L'Italien et al. 2007; Saddichha et al. 2007; Srisurapanont et al. 2007; De Hert et al. 2008b; Meyer et al. 2008; Kim et al. 2010; Kraemer et al. 2010]. The results were hardly comparable in this field as incidence rates were calculated for various time periods, from 6 weeks up to 1 year. One of these studies referred to a population of 30 young drug-naïve women who were medicated with an antipsychotic agent for 6 weeks, and provided MetS incidence estimates at the beginning and the end of the trial period (3.33-31.81\%) [Saddichha et al. 2007]. This was a clear and rare example of how quickly and extensively MetS can develop as a response to antipsychotic administration in an otherwise healthy population.

\section{Framingham 10-year risk}

A few studies calculated Framingham 10-year cardiovascular risk rates for their patients [Cohn et al. 2004; Correll et al. 2006, 2008; Bobes et al. 2007; Rejas et al. 2008; Yazici et al. 2011]. When controls were also included, study populations scored substantially higher [Cohn et al. 2004]. Men scored higher than women in all studies that calculated rates according to sex. When cardiovascular risk rates were calculated for age clusters, the highest values were observed in the fifth and sixth decade of life.

\section{Findings from other reviews}

Our findings build on those from other reviews: first it is clear that there is an association between metabolic risk factors and antipsychotic use; second it seems that antipsychotic use alone is not sufficient to explain the increased metabolic risk seen in schizophrenia.

In a thorough systematic review of atypical antipsychotics and weight gain, Taylor and colleagues discussed a total of 74 papers ( 6 on amisulpride, 27 on clozapine, 8 on olanzapine, 7 on quetiapine, 13 on risperidone, 7 on sertindole, 3 on ziprasidone and 3 on zotepine) [Taylor and McAskill, 2000]. They concluded that with the exception of ziprasidone, all medications have been associated with weight gain; however aripiprazole was not included in this review.

Jin and colleagues reviewed studies on the effect of atypical antipsychotics (clozapine, risperidone, olanzapine, quetiapine, ziprasidone and aripiprazole) on glucose dysregulation [Jin et al. 2004]. They included four extensive case series and summaries, 13 epidemiological studies from prescription and drug safety monitoring databases and 10 clinical studies on glucose intolerance and atypical antipsychotics. Despite individual differences noted among various antipsychotics, they noted that patients with known risk factors for type II diabetes mellitus, such as ethnicity, firstdegree family history of diabetes mellitus and baseline obesity, appear to be at increased risk for the development of glucose dysregulation during treatment.

Meyer and Koro reviewed 48 studies and focused on the effects of FGAs and SGAs on serum lipids [Meyer and Koro, 2004]. They stated that highpotency conventional antipsychotics (haloperidol) and some atypicals (ziprasidone, risperidone and aripiprazole) are associated with lower risk of dyslipidaemia, whilst low-potency conventional antipsychotics (chlorpromazine, thioridazine) and some other atypicals (quetiapine, olanzapine and clozapine) are related to higher risk of dyslipidaemia.

Newcomer, in a massive and comprehensive literature review, gathered data from more than 200 studies on the metabolic effects of atypical antipsychotics, with special focus on weight gain and glucose and lipid dysregulation [Newcomer, 2005]. He extensively discussed eight antipsychotic medications, specifically clozapine, olanzapine, risperidone, quetiapine, zotepine, amisulpride, ziprasidone and aripiprazole, and provided a detailed account of the metabolic profile of each. Clozapine and olanzapine were associated with the highest risk for causing clinically significant weight gain; risperidone, quetiapine, amisulpiride and zotepine a moderate risk; ziprasidone and aripiprazole a lower risk. $\mathrm{He}$ also noted that this ranking reflected the relative risk for insulin resistance, dyslipidaemia and hyperglycaemia.

In a systematic review and meta-analysis Smith and colleagues compared FGAs and SGAs with regards to their risk for type II diabetes mellitus [Smith et al. 2008]. The atypical antipsychotics included in this review (clozapine, olanzapine, risperidone and quetiapine) appeared to have a small increased risk only for development of diabetes compared with typical antipsychotics. 
Simon and colleagues attempted to investigate whether weight gain and metabolic side effects of atypical antipsychotics are dose dependent in a systematic review of 45 original articles [Simon et al. 2009]. Among the medications included (clozapine, olanzapine, risperidone, quetiapine, ziprasidone, sertindole, amisulpiride and aripiprazole) a dose-response relationship between serum concentrations and metabolic outcomes was suggested only for clozapine and olanzapine, although the association between daily dose and metabolic measures was not clear.

Meyer and colleagues summarized the information available about MetS in patients with schizophrenia and proposed mechanisms for the increased prevalence of MetS in this population [Meyer et al. 2005a]. They suggested that the vulnerability to developing MetS among patients with schizophrenia derives from the concept that the phenotype of MetS resembles that of Cushing's disease, and thus is related to an inherent dysregulation of the hypothalamo-pituitaryadrenal (HPA) axis.

Meyer and Stahl returned with a second review in which they focused on the prevalence of MetS in schizophrenia, presenting data from the latest studies [Meyer and Stahl, 2009]. They also attempted to address the great debate about whether the development of MetS is an environmental epiphenomenon related to health habits seen in schizophrenia, or a fundamental part of this disease. Special emphasis was placed on the importance of the fasting serum triglyceride to HDL ratio in predicting insulin resistance better than fasting serum glucose. The authors also discussed the issue of metabolic monitoring in patients with schizophrenia and made appropriate reference to a number of behavioural and pharmacological interventions. They concluded that because of lifestyle, disease and medication effects, patients with schizophrenia have significant risk for cardio-metabolic disease. They also recommended routine monitoring, preferential use of metabolically neutral antipsychotics, antidiabetic medication and lifestyle education as ways to minimize risk.

In a review of MetS and psychiatric illness, Mendelson emphasized the pathophysiological links between the development of MetS and the emergence of psychotic symptoms in schizophrenia by investigating the role of asymmetrical dimethylarginine (ADMA), homocysteine, s-adenosylmethionine (SAMe) and N-methylD-aspartate receptors (NMDAR) [Mendelson, 2008]. He highlighted the presence of increased levels of ADMA as a common feature between MetS and schizophrenia. ADMA is an endogenous inhibitor of nitric oxide, which is a major intracellular mediator of NMDAR activation. Thus ADMA might contribute to decreases in NMDAR activity that, in turn, may increase the psychotic symptoms in schizophrenia.

Lindley and colleagues provided a detailed account of the insulin-resistance syndrome in schizophrenia, referring to a number of endogenous and exogenous factors [Lindley et al. 2008]. They emphasized the strong links between insulin resistance and lifestyle characteristics (including weight, diet, exercise, energy expenditure) and discussed the role of stress and HPA axis dysregulation in antipsychotic-induced metabolic disturbances. They also noticed that significantly higher fasting glucose levels are observed in drugnaïve patients with schizophrenia, suggesting an integral role of insulin resistance in this disorder. Following this path of thought they introduced the hypothesis that insulin resistance shares genetic risk factors with schizophrenia and mood disorders.

Among the most comprehensive reviews is without doubt that of Leucht and colleagues [Leucht et al. 2007a, 2007b]. In their impressive work, the authors performed a pedantic review of 52 original articles since 1919, the majority of which describe individual features of MetS in patients with schizophrenia. This is definitely one of the most systematic attempts to present both the extent and the nature of this condition. The authors provided a detailed account of numerous estimations of the prevalence of MetS in schizophrenia, showing almost unanimously increased rates compared with the general population.

In a review, De Hert and colleagues summarized all estimates of the prevalence and incidence of MetS in schizophrenia from 2003 onwards [De Hert et al. 2009]. They also provided suggestions for screening and monitoring of MetS in patients with schizophrenia and emphasized the importance of a multidisciplinary assessment of psychiatric and physical conditions. The authors returned in 2012 with two more meta-analyses on metabolic and cardiovascular adverse effects associated with antipsychotic drugs [De Hert et al. 
2012a, 2012b]. They concluded that the potential of SGAs to induce or trigger metabolic dysregulation, including type II diabetes mellitus and MetS, is firmly established. They ranked SGAs from high to low in terms of cardiovascular adverse effects as follows: clozapine $=$ olanzapine $>$ quetiapine $\geq$ risperidone $=$ paliperidone $>$ amisulpride $>$ aripiprazole $\geq$ ziprasidone. They noted that, for the FGAs, the low-potency agents have the highest potential and the high-potency agents the lowest potential to induce metabolic dysfunction. The risk profiles of the FGAs are comparable to those of the highand low-risk SGAs. They also recommended regular monitoring as part of the management of patients receiving antipsychotic drugs.

The most recent meta-analyses on this topic come from Mitchell and colleagues [Mitchell et al. $2011,2012 \mathrm{~b}$ ]. The authors provided a very comprehensive review of prevalence and predictors of MetS in adults with schizophrenia and related disorders, accounting for subgroup differences. The overall rate of MetS was calculated at 32.5\% and they were only minor differences according to different definitions, treatment setting, country of origin and no appreciable differences between men and women. Older age had a modest influence on the rate of MetS, while the duration of illness had the strongest influence. Waist circumference proved to be the most useful measure in predicting high rates of MetS. The highest rates of MetS were seen in those prescribed clozapine $(51.9 \%)$ and the lowest rates in those who were unmedicated $(20.2 \%)$.

There was a significantly lower cardiovascular risk in early compared with chronic schizophrenia. Diabetes and prediabetes appeared uncommon in the early stages, especially in unmedicated patients.

Overall all the reviews already published agree that MetS is increasingly present in patients with schizophrenia. Most authors emphasized the importance of extrinsic factors (antipsychotic medication, increased calorie intake, sedentary lifestyle) in its development, however the concept of intrinsic factors being implicated (genetic links between schizophrenia and diabetes) is also supported.

\section{Discussion}

Summarizing the findings

MetS is over represented in SMI, and patients with schizophrenia usually have a twofold (or more) risk of developing it compared with the general population. Its prevalence varies largely (3.9-68\%) across various countries and studies: it is least likely in young, unmedicated, drug-naïve patients and most likely to be seen in chronically ill and long-term medicated patients. Women tend to present with increased rates of MetS compared with men, and some ethnic groups, such as black Africans and Hispanics show a possible predisposition to the condition. Prevalence of MetS in patients with schizophrenia generally increases with age, as in the general population, and the highest rates for Framingham 10-year cardiovascular risk are observed in men and in the fifth and sixth decades of life. It is of note that women tend to have higher rates of MetS and men higher rates of Framingham 10-year cardiovascular risk; at first glance, this looks like a paradoxical observation. However, we should consider that MetS reflects the current metabolic profile of an individual (based on a variety of physiological measures) while the Framingham 10-year risk describes the likelihood of a cardiovascular event in the future and is calculated by using metabolic measures (HDL, total cholesterol, systolic blood pressure) and epidemiological data (age, smoking status, treatment for hypertension) [D'Agostino et al. 2008]. So men are still at higher risk of developing cardiovascular disease (possibly due to a combination of unhealthy life habits, lack of medical input and abnormal metabolic status) while women show a higher point prevalence of MetS, which can reflect a gender-specific metabolic profile. SGAs, high-potency antipsychotic agents (both FGAs and SGAs) and polypharmacy are risk factors for the development of MetS. Other risk factors include the length of exposure to psychotic illness and lifestyle habits, such as smoking. The incidence of MetS in schizophrenia is hard to calculate but it can develop quickly, even within 6 weeks of starting antipsychotic medication.

\section{Directions for future research: the genetics}

In this review, we emphasized the importance of extrinsic factors in the development of MetS. However, we cannot remain oblivious to the large and growing amount of evidence suggesting that MetS also has a strong genetic component. Numerous genome wide association studies (GWAS) have identified various single nucleotide polymorphisms (SNPs) associated with the different components of MetS. These SNPs are usually found in the vicinity of genes that play various 
roles in one or more metabolic pathways. Relevant literature provides a wealth of evidence:

(1) BMI: in a large GWAS of 249,706 individuals, Speliotes identified 18 new loci associated with BMI, in addition to 14 SNPs previously associated with BMI and waist and weight measurements [Speliotes, 2010].

(2) Hypertension, blood pressure: two GWAS of 34,433 and 29,136 individuals respectively identified a total of 38 loci associated with hypertension or blood pressure [Levy et al. 2009; Newton-Cheh et al. 2009].

(3) Type II diabetes: Zeggini and colleagues reviewed the literature on loci associated with type II diabetes and identified six additional susceptibility loci in a largescale replication study of 53,795 individuals [Zeggini et al. 2008].

(4) Dyslipidaemia: a large-scale GWAS of more than 100,000 individuals identified 95 loci associated with both normal variation in blood lipid traits and extreme blood lipid phenotypes [Teslovich et al. 2010]. Two more studies of 5414 and 132 individuals respectively identified SNPs associated with blood cholesterol levels and hypertriglyceridaemia [Kathiresan et al. 2008; Wang et al. 2008].

(5) Recently a number of GWAS also focused on MetS as an entity. Kraja and colleagues in their analysis including 22162 samples identified 29 common variants associated with MetS or a pair of its traits [Kraja et al. 2011]. Zabaneh and Balding analysed 4794 samples and identified nine loci associated with the development of MetS in Asian men, not substantially different from MetS determinants in other populations [Zabaneh and Balding, 2010].

(6) A number of studies have also implicated several genes in the development of MetS. Polymorphic variants of the gene encoding 5,10-methylenetrahydrofolate reductase (MTHFR gene) appear to infer an increased risk for metabolic abnormalities, especially in response to antipsychotic medication [Van Winkel et al. 2010; Kuzman and Mueller, 2012]. A polymorphism of the gene encoding the adrenergic $\alpha 1 \mathrm{~A}$ receptor (ADRA1A gene) is found to be a risk factor for severe metabolic abnormalities [Cheng et al. 2012]. Similarly, another polymorphism of the gene encoding serotonin $2 \mathrm{C}$ receptor (HTR2C gene) is also associated with increased risk of MetS in patients taking antipsychotics, particularly those using clozapine or risperidone [Mulder et al. 2007, 2009]. Polymorphic variants of the cannabinoid type 1 receptor gene (CNR1 gene) are associated with obesity-related phenotypes in women [Milewicz et al. 2010]. Finally, variations in genes implicated in circadian regulation (clock genes) may be important in the regulation of metabolic parameters in schizophrenia, during long-term treatment with SGAs [Moons et al. 2011].

These studies indicate that the cardiovascular risk factors associated with MetS are to a certain extent genetically determined. This means that there is likely a genetic aspect to the cardiovascular risk seen in people with schizophrenia. Future research should address this emerging concept of the genetic predisposition to develop MetS in schizophrenia. Thus we could potentially develop diagnostic tools that will inform us of the risks carried by a particular individual of developing MetS, even before the initiation of antipsychotic medication. This would allow appropriate medication choices to be made early in the course of illness and adequate health interventions to be implemented sooner rather than later. Hopefully more research in the future will be able to clarify these complex metabolic pathways and provide proactive management of all these metabolic disturbances.

\section{The importance of monitoring for the \\ prevention of metabolic syndrome}

The cornerstone of early detection and effective management of MetS in patients with schizophrenia is comprehensive monitoring, and a variety of guidelines provide structured schedules for this. Despite the introduction of guidelines for metabolic screening in schizophrenia, metabolic monitoring in routine clinical practice is still low. In their impressive meta-analysis of 48 studies, Mitchell and colleagues reviewed changes in monitoring screening of patients receiving antipsychotics before and after the implementation of relevant guidelines [Mitchell et al. 2012a]. They concluded that although guidelines can increase monitoring, most patients still do not receive 
adequate testing. Similar results come from another group of researchers who found that glucose and lipid screening is underutilized in patients starting on SGAs [Morrato et al. 2008], and the introduction of the American Diabetes Association's Consensus Statement on antipsychotic drugs and diabetes was not associated with an increase in screening rates [Morrato et al. 2009].

Apart from the basic features of MetS (BMI, fasting plasma glucose, fasting plasma lipids, blood pressure), other tests such as electrocardiography and routine blood tests (urea and electrolytes, liver function tests, full blood count, prolactin levels) can complement the laboratory and physical checks of patients with schizophrenia, especially those in receipt of antipsychotic medication. A medical and family history should also be included in this monitoring, and in most cases it is meaningful to accompany the whole process with regular advice on healthy living. The frequency of monitoring can vary and be adapted to the individual needs of patients. However, it is more important that this process is incorporated into regular psychiatric follow up. The findings that certain ethnic groups, female sex, family history and type of medication all increase the risk of developing MetS can be used by practitioners to identify and target certain individuals who are likely to be at greater risk of life-threatening cardiovascular disease should they develop schizophrenia.

\section{Funding}

This research received no specific grant from any funding agency in the public, commercial, or notfor-profit sectors

\section{Conflict of interest statement}

The authors declare that there is no conflict of interest.

\section{References}

Alberti, K., Eckel, R., Grundy, S., Zimmet, P., Cleeman, J., Donato, K. et al. (2009) Harmonizing the metabolic syndrome: a joint interim statement of the International Diabetes Federation Task Force on Epidemiology and Prevention; National Heart, Lung, and Blood Institute; American Heart Association; World Heart Federation; International Atherosclerosis Society; and International Association for the Study of Obesity. Circulation 120: 1640-1645.
Almeras, N., Despres, J., Villeneuve, J., Demers, M., Roy, M., Cadrin, C. et al. (2004) Development of an atherogenic metabolic risk factor profile associated with the use of atypical antipsychotics. $\mathcal{F}$ Clin Psychiatry 65: 557-564.

Attux, C., Quintana, M. and Chaves, A. (2007) Weight gain, dyslipidemia and altered parameters for metabolic syndrome on first episode psychotic patients after six-month follow-up. Rev Bras Psiquiatr 29: 346-349.

Basu, R., Brar, J., Chengappa, K., John, V., Parepally, H., Gershon, S. et al. (2004) The prevalence of the metabolic syndrome in patients with schizoaffective disorder - bipolar subtype. Bipolar Disord 6: 314-318.

Bobes, J., Arango, C., Aranda, P., Carmena, R., Garcia-Garcia, M. and Rejas, J. (2007)

Cardiovascular and metabolic risk in outpatients with schizophrenia treated with antipsychotics: results of the Clamors Study. Schizophr Res 90: 162-173.

Boke, O., Aker, S., Sarisoy, G., Saricicek, E. and Sahin, A. (2008) Prevalence of metabolic syndrome among inpatients with schizophrenia. Int $\mathcal{F}$ Psychiatry Med 38: 103-112.

Cerit, C., Ozten, E. and Yildiz, M. (2008) [The prevalence of metabolic syndrome and related factors in patients with schizophrenia]. Turk Psikiyatri Derg 19: 124-132.

Cheng, C., Chiu, H., Loh, E., Chan, C., Hwu, T., Liu, Y. et al. (2012) Association of the ADRA1A gene and the severity of metabolic abnormalities in patients with schizophrenia. Prog Neuropsychopharmacol Biol Psychiatry 36: 205-210.

Cohn, T., Prud'homme, D., Streiner, D., Kameh, H. and Remington, G. (2004) Characterizing coronary heart disease risk in chronic schizophrenia: high prevalence of the metabolic syndrome. Can $\mathcal{F}$ Psychiatry 49: 753-760.

Cornier, M., Dabelea, D., Hernandez, T., Lindstrom, R., Steig, A., Stob, N. et al. (2008) The metabolic syndrome. Endocr Rev 29: 777-822.

Correll, C., Frederickson, A., Kane, J. and Manu, P. (2006) Metabolic syndrome and the risk of coronary heart disease in 367 patients treated with secondgeneration antipsychotic drugs. $\mathcal{F}$ Clin Psychiatry 67: 575-583.

Correll, C., Frederickson, A., Kane, J. and Manu, P. (2007) Does antipsychotic polypharmacy increase the risk for metabolic syndrome? Schizophr Res 89: 91-100.

Correll, C., Frederickson, A., Kane, J. and Manu, P. (2008) Equally increased risk for metabolic syndrome in patients with bipolar disorder and schizophrenia 
treated with second-generation antipsychotics. Bipolar Disord 10: 788-797.

Curtis, J., Henry, C., Watkins, A., Newall, H., Samaras, K. and Ward, P. (2011) Metabolic abnormalities in an early psychosis service: a retrospective, naturalistic cross-sectional study. Early Interv Psychiatry 5: 108-114.

D’Agostino, R., Vasan, R., Pencina, M., Wolf, P., Cobain, M., Massaro, J. et al. (2008) General cardiovascular risk profile for use in primary care: the Framingham Heart Study. Circulation 117: 743-753.

Danaei, G., Finucane, M., Lin, J., Singh, G., Paciorek, C., Cowan, M. et al. (2011) National, regional, and global trends in systolic blood pressure since 1980: systematic analysis of health examination surveys and epidemiological studies with 786 countryyears and 5.4 million participants. Lancet 377 : 568-577.

De Hert, M., Detraux, J., Van Winkel, R., Yu, W. and Correll, C. (2012a) Metabolic and cardiovascular adverse effects associated with antipsychotic drugs. Nat Rev Endocrinol 8: 114-126.

De Hert, M., Falissard, B., Mauri, M., Shaw, K. and Wetterling, T. (2008a) Epidemiological study for the evaluation of metabolic disorders in patients with schizophrenia: the METEOR study. Eur Neuropsychopharmacol 18: S444-S445.

De Hert, M., Hanssens, L., Wampers, M., Van Winkel, R., Van Eyck, D., Scheen, A. et al. (2007) Prevalence and incidence rates of metabolic abnormalities and diabetes in a prospective study of patients treated with second-generation antipsychotics. Schizophr Bull 33: 560.

De Hert, M., Mauri, M., Shaw, K., Wetterling, T., Doble, A., Giudicelli, A. et al. (2010) The METEOR study of diabetes and other metabolic disorders in patients with schizophrenia treated with antipsychotic drugs. I. Methodology. Int $\mathcal{F}$ Methods Psychiatr Res 19: 195-210.

De Hert, M., Schreurs, V., Sweers, K., Van Eyck, D., Hanssens, L., Sinko, S. et al. (2008b) Typical and atypical antipsychotics differentially affect long-term incidence rates of the metabolic syndrome in firstepisode patients with schizophrenia: a retrospective chart review. Schizophr Res 101: 295-303.

De Hert, M., Schreurs, V., Vancampfort, D. and Van Winkel, R. (2009) Metabolic syndrome in people with schizophrenia: a review. World Psychiatry 8: 15-22.

De Hert, M., Van Winkel, R., Van Eyck, D., Hanssens, L., Wampers, M., Scheen, A. et al. (2006a) Prevalence of diabetes, metabolic syndrome and metabolic abnormalities in schizophrenia over the course of the illness: a cross-sectional study. Clin Pract Epidemiol Ment Health 2: 14.
De Hert, M., Van Winkel, R., Van Eyck, D., Hanssens, L., Wampers, M., Scheen, A. et al. (2006b) Prevalence of the metabolic syndrome in patients with schizophrenia treated with antipsychotic medication. Schizophr Res 83: 87-93.

De Hert, M., Yu, W., Detraux, J., Sweers, K., Van Winkel, R. and Correll, C. (2012b) Body weight and metabolic adverse effects of asenapine, iloperidone, lurasidone and paliperidone in the treatment of schizophrenia and bipolar disorder: a systematic review and exploratory meta-analysis. CNS Drugs 26: 733-759.

Farzadfar, F., Finucane, M., Danaei, G., Pelizzari, P., Cowan, M., Paciorek, C. et al. (2011) National, regional, and global trends in serum total cholesterol since 1980: systematic analysis of health examination surveys and epidemiological studies with 321 countryyears and 3.0 million participants. Lancet 377 : $578-586$.

Fernandez-Egea, E., Bernardo, M., Donner, T., Conget, I., Parellada, E., Justicia, A. et al. (2009) Metabolic profile of antipsychotic-naive individuals with non-affective psychosis. Br F Psychiatry 194: 434-438.

Fernandez-Egea, E., Bernardo, M., Parellada, E., Justicia, A., Garcia-Rizo, C., Esmatjes, E. et al. (2008a) Glucose abnormalities in the siblings of people with schizophrenia. Schizophr Res 103: 110-113.

Fernandez-Egea, E., Miller, B., Bernardo, M., Donner, T. and Kirkpatrick, B. (2008b) Parental history of type 2 diabetes in patients with nonaffective psychosis. Schizophr Res 98: 302-306.

Finucane, M., Stevens, G., Cowan, M., Danaei, G., Lin, J., Paciorek, C. et al. (2011) National, regional, and global trends in body-mass index since 1980: systematic analysis of health examination surveys and epidemiological studies with 960 country-years and 9.1 million participants. Lancet 377: 557-567.

Hagg, S., Lindblom, Y., Mjorndal, T. and Adolfsson, R. (2006) High prevalence of the metabolic syndrome among a Swedish cohort of patients with schizophrenia. Int Clin Psychopharmacol 21: 93-98.

Hansen, T., Ingason, A., Djurovic, S., Melle, I., Fenger, M., Gustafsson, O. et al. (2011) At-risk variant in TCF712 for type II diabetes increases risk of schizophrenia. Biol Psychiatry 70: 59-63.

Heiskanen, T., Niskanen, L., Lyytikainen, R., Saarinen, P. and Hintikka, J. (2003) Metabolic syndrome in patients with schizophrenia. $\mathcal{F}$ Clin Psychiatry 64: 575-579.

Huang, M., Lu, M., Tsai, C., Chen, P., Chiu, C., Jian, D. et al. (2009) Prevalence of metabolic 
syndrome among patients with schizophrenia or schizoaffective disorder in Taiwan. Acta Psychiatr Scand 120: 274-280.

International Diabetes Federation (2006) The IDF Consensus Worldwide Definition of the Metabolic Syndrome. Brussels: International Diabetes Federation.

Jin, H., Meyer, J. and Jeste, D. (2004) Atypical antipsychotics and glucose dysregulation: a systematic review. Schizophr Res 71: 195-212.

Kang, S., Kim, K., Kang, G., Lee, K., Kim, K., Soh, M. et al. (2011) Cross-sectional prevalence of metabolic syndrome in korean patients with schizophrenia. Schizophr Res 128: 179-181.

Kathiresan, S., Melander, O., Anevski, D., Guiducci, C., Burtt, N., Roos, C. et al. (2008) Polymorphisms associated with cholesterol and risk of cardiovascular events. $N$ Engl $\mathcal{F}$ Med 358: 1240 .

Kato, M., Currier, M., Gomez, C., Hall, L. and Gonzalez-Blanco, M. (2004) Prevalence of metabolic syndrome in Hispanic and non-Hispanic patients with schizophrenia. Prim Care Companion 7 Clin Psychiatry 6: 74-77.

Kim, E., Lee, N., Kim, S., Jung, D. and Ahn, Y. (2010) Change in the rate of metabolic syndrome in patients with schizophrenia and bipolar disorder in the course of treatment. Bipolar Disord 12: 31-31.

Koponen, H., Alaraisanen, A., Saari, K., Pelkonen, O., Huikuri, H., Raatikainen, M. et al. (2008)

Schizophrenia and sudden cardiac death - a review. Nord F Psychiatry 62: 342-345.

Koponen, H., Hakko, H., Saari, K., Lindeman, S., Karvonen, K., Isohanni, M. et al. (2010) The prevalence and predictive value of individual criteria for metabolic syndrome in schizophrenia: a northern Finland 1966 birth cohort study. World $\mathcal{F}$ Biol Psychiatry 11: 262-267.

Kraemer, S., Minarzyk, A., Forst, T., Kopf, D. and Hundemer, H. (2011) Prevalence of metabolic syndrome in patients with schizophrenia, and metabolic changes after 3 months of treatment with antipsychotics - results from a German observational study. BMC Psychiatry 11: 173.

Kraemer, S., Minarzyk, A., Hundemer, H., Forst, T. and Kopf, D. (2010) Sex-specific differences in the prevalence of metabolic syndrome components in patients with schizophrenia - results from a German observational study. F Psychopharmacol 24: A45.

Kraja, A., Vaidya, D., Pankow, J., Goodarzi, M., Assimes, T., Kullo, I. et al. (2011) A bivariate genome-wide approach to metabolic syndrome: STAMPEED consortium. Diabetes 60: 1329-1339.
Kuzman, M. and Mueller, D. (2012) Association of the MTHFR gene with antipsychotic-induced metabolic abnormalities in patients with schizophrenia. Pharmacogenomics 13: 843-846.

Lamberti, J., Olson, D., Crilly, J., Olivares, T., Williams, G., Tu, X. et al. (2006) Prevalence of the metabolic syndrome among patients receiving clozapine. Am f Psychiatry 163: 1273-1276.

Lee, N., Kim, S., Jung, D., Kim, E., Yu, H., Sung, K. et al. (2011) The prevalence of metabolic syndrome in Korean patients with schizophrenia receiving a monotherapy with aripiprazole, olanzapine or risperidone. Prog Neuropsychopharmacol Biol Psychiatry 35: 1273-1278.

Lee, N., Yu, H., Jung, D., Kim, S., Kim, Y. and Ahn, Y. (2009) Higher prevalence of metabolic syndrome in patient with bipolar disorder and schizophrenia in Korean population. Eur Neuropsychopharmacol 19: S540.

Leucht, S., Burkard, T., Henderson, J., Maj, M. and Sartorius, N. (2007a) Physical illness and schizophrenia: a review of the literature. Acta Psychiatr Scand 116: 317-333.

Leucht, S., Burkard, T., Henderson, J., Maj, M. and Sartorius, N. (2007b) Physical Illness and Schizophrenia: A Review of the Evidence. Cambridge: Cambridge Universtiy Press.

Levy, D., Ehret, G., Rice, K., Verwoert, G., Launer, L., Dehghan, A. et al. (2009) Genome-wide association study of blood pressure and hypertension. Nat Genet 41: 677-687.

Lindley, S., Schroder, C., Hara, R. and Fazio, S. (2008) Insulin resistance in schizophrenia, major depression, and bipolar disorder. In: Frosch, W. (ed), Insulin Resistance Syndrome and Neuropsychiatric Disease. New York: Informa Healthcare.

L'Italien, G., Casey, D., Kan, H., Carson, W. and Marcus, R. (2007) Comparison of metabolic syndrome incidence among schizophrenia patients treated with aripiprazole versus olanzapine or placebo. f Clin Psychiatry 68: 1510-1516.

Mackin, P., Bishop, D., Watkinson, H., Gallagher, P. and Ferrier, I. (2007) Metabolic disease and cardiovascular risk in people treated with antipsychotics in the community. Br F Psychiatry 191: 23-29.

Maudsley, H. (1979) The Pathology of Mind. 3rd ed. London: Macmillan.

McEvoy, J., Meyer, J. and Goff, K. (2005) Prevalence of the metabolic syndrome in patients with schizophrenia: baseline results from the Clinical Antipsychotic Trials of Intervention Effectiveness (CATIE) schizophrenia trial and comparison with national estimates from NHANES III. Schizophr Res 80: 19-32. 
Mendelson, S.D. (2008) Metabolic Syndrome and Psychiatric Illness. In: Mendelson, S.D., (ed), Metabolic Syndrome and Psychiatric Illness: Interactions, Pathophysiology, Assessment and Treatment. Academic Press: London.

Meyer, J., Davis, V., Goff, D., Mcevoy, J., Nasrallah, H., Davis, S. et al. (2008) Change in metabolic syndrome parameters with antipsychotic treatment in the CATIE schizophrenia trial: prospective data from phase 1. Schizophr Res 101: 273-286.

Meyer, J. and Koro, C. (2004) The effects of antipsychotic therapy on serum lipids: a comprehensive review. Schizophr Res 70: 1-17.

Meyer, J., Koro, C. and L'Italien, G. (2005a) The metabolic syndrome and schizophrenia: a review. Int Rev Psychiatry 17: 173-180.

Meyer, J., Loh, C., Leckband, S., Boyd, J., Wirshing, W., Pierre, J. et al. (2006) Prevalence of the metabolic syndrome in veterans with schizophrenia. F Psychiatr Pract 12: 5-10.

Meyer, J., Nasrallah, H. and McEvoy, J. (2005b) The Clinical Anti-Psychotic Trials of Intervention Effectiveness (CATIE) schizophrenia trial: clinical comparison of subgroups with and without the metabolic syndrome. Schizophr Res 80: 9-18.

Meyer, J. and Stahl, S. (2009) The metabolic syndrome and schizophrenia. Acta Psychiatr Scand 119: 4-14.

Milewicz, A., Tworowska-Bardzińska, U., Jędrzejuk, D., Lwow, F., Dunajska, K., Łaczmański, Ł. et al. (2010) Are endocannabinoid type 1 receptor gene (CNR1) polymorphisms associated with obesity and metabolic syndrome in postmenopausal Polish women. Int $\mathcal{F}$ Obes 35: 373-377.

Mitchell, A., Delaffon, V., Vancampfort, D., Correll, C. and De Hert, M. (2012a) Guideline concordant monitoring of metabolic risk in people treated with antipsychotic medication: systematic review and metaanalysis of screening practices. Psychol Med 42: 125-127.

Mitchell, A., Vancampfort, D., De Herdt, A., Yu, W. and De Hert, M. (2012b) Is the prevalence of metabolic syndrome and metabolic abnormalities increased in early schizophrenia? A comparative metaanalysis of first episode, untreated and treated patients. Schizophr Bull 27 August (Epub ahead of print).

Mitchell, A., Vancampfort, D., Sweers, K., Van Winkel, R., Yu, W. and De Hert, M. (2011)

Prevalence of metabolic syndrome and metabolic abnormalities in schizophrenia and related disorders a systematic review and meta-analysis. Schizophr Bull 29 December (Epub ahead of print).

Moons, T., Claes, S., Martens, G., Peuskens, J., Van Loo, K., Van Schijndel, J. et al. (2011) Clock genes and body composition in patients with schizophrenia under treatment with antipsychotic drugs. Schizophr Res 125: 187-193.

Morrato, E., Newcomer, J., Allen, R. and Valuck, R. (2008) Prevalence of baseline serum glucose and lipid testing in users of second-generation antipsychotic drugs: a retrospective, population-based study of Medicaid claims data. F Clin Psychiatry 69: 316-322.

Morrato, E., Newcomer, J., Kamat, S., Baser, O., Harnett, J. and Cuffel, B. (2009) Metabolic screening after the American Diabetes Association's consensus statement on antipsychotic drugs and diabetes. Diabetes Care 32: 1037-1042.

Mozumdar, A. and Liguori, G. (2011) Persistent increase of prevalence of metabolic syndrome among US adults: NHANES III to NHANES 1999-2006. Diabetes Care 34: 216-219.

Mulder, H., Cohen, D., Scheffer, H., Gispen-De Wied, C., Arends, J., Wilmink, F. et al. (2009) HTR2C gene polymorphisms and the metabolic syndrome in patients with schizophrenia: a replication study. F Clin Psychopharmacol 29: 16-20.

Mulder, H., Franke, B., Van der-Beek van der, A., Arends, J., Wilmink, F., Scheffer, H. et al. (2007) The association between HTR2C gene polymorphisms and the metabolic syndrome in patients with schizophrenia. $\mathcal{F}$ Clin Psychopharmacol 27: 338-343.

Newcomer, J. (2005) Second-generation atypical antipsychotics and metabolic effects. a comprehensive literature review. CNS Drugs 19: 1-93.

Newton-Cheh, C., Johnson, T., Gateva, V., Tobin, M., Bochud, M., Coin, L. et al. (2009) Genome-wide association study identifies eight loci associated with blood pressure. Nat Genet 41: 666-676.

Padmavati, R., McCreadie, R. and Tirupati, S. (2010) Low prevalence of obesity and metabolic syndrome in never-treated chronic schizophrenia. Schizophr Res 121: 199-202.

Rejas, J., Bobes, J., Arango, C., Aranda, P., Carmena, R. and Garcia-Garcia, M. (2008) Concordance of standard and modified NCEP ATP III criteria for identification of metabolic syndrome in outpatients with schizophrenia treated with antipsychotics: a corollary from the CLAMORS study. Schizophr Res 99: 23-28.

Rezaei, O., Khodaie-Ardakani, M., Mandegar, M., Dogmehchi, E. and Goodarzynejad, H. (2009)

Prevalence of metabolic syndrome among an Iranian cohort of inpatients with schizophrenia. Int $\mathcal{F}$ Psychiatry Med 39: 451-462.

Saari, K., Lindeman, S., Viilo, K., Isohanni, M., Jarvelin, M., Lauren, L. et al. (2005) A 4-fold risk of metabolic syndrome in patients with schizophrenia: 
the Northern Finland 1966 birth cohort study. F Clin Psychiatry 66: 559-563.

Saddichha, S., Ameen, S. and Akhtar, S. (2007) Incidence of new onset metabolic syndrome with atypical antipsychotics in first episode schizophrenia: a six-week prospective study in Indian female patients. Schizophr Res 95: 247.

Saddichha, S., Manjunatha, N., Ameen, S. and Akhtar, S. (2008) Metabolic syndrome in first episode schizophrenia - a randomized double-blind controlled, short-term prospective study. Schizophr Res 101: 266-272.

Saha, S., Chant, D. and McGrath, J. (2007) A systematic review of mortality in schizophrenia - is the differential mortality gap worsening over time? Arch Gen Psychiatry 64: 1123-1131.

Schorr, S., Slooff, C., Bruggeman, R. and Taxis, K. (2009) Prevalence of metabolic syndrome in patients with psychotic disorders in the Netherlands. $f$ Clin Psychopharmacol 29: 399-402.

Sicras-Mainar, A., Blanca-Tamayo, M., RejasGutierrez, J. and Navarro-Artieda, R. (2008) Metabolic syndrome in outpatients receiving antipsychotic therapy in routine clinical practice: a cross-sectional assessment of a primary health care database. Eur Psychiatry 23: 100-108.

Simon, V., Van Winkel, R. and De Hert, M. (2009) Are weight gain and metabolic side effects of atypical antipsychotics dose dependent? A literature review. f Clin Psychiatry 70: 1041-1050.

Smith, M., Hopkins, D., Peveler, R., Holt, R., Woodward, M. and Ismail, K. (2008) First- v. second-generation antipsychotics and risk for diabetes in schizophrenia: systematic review and meta-analysis. Br F Psychiatry 192: 406-411.

Speliotes, E. (2010) Association analyses of 249,796 individuals reveal 18 new loci associated with body mass index. Nat Genet 42: 937-950.

Srisurapanont, M., Likhitsathian, S., Boonyanaruthee, V., Charnsilp, C. and Jarusuraisin, N. (2007) Metabolic syndrome in Thai schizophrenic patients: a naturalistic one-year follow-up study. BMC Psychiatry 7: 14.

Straker, D., Correll, C., Kramer-Ginsberg, E., Abdulhamid, N., Koshy, F., Rubens, E. et al. (2005) Cost-effective screening for the metabolic syndrome in patients treated with second-generation antipsychotic medications. Am f Psychiatry 162: 1217-1221.

Subashini, R., Deepa, M., Padmavati, R., Thara, R. and Mohan, V. (2011) Prevalence of diabetes, obesity, and metabolic syndrome in subjects with and without schizophrenia (CURES-104). F Postgrad Med 57: 272-277.
Sugawara, N., Yasui-Furukori, N., Sato, Y., Kishida, I., Yamashita, H., Saito, M. et al. (2011) Comparison of prevalence of metabolic syndrome in hospital and community-based Japanese patients with schizophrenia. Ann Gen Psychiatry 10: 21.

Sugawara, N., Yasui-Furukori, N., Sato, Y., Umeda, T., Kishida, I., Yamashita, H. et al. (2010) Prevalence of metabolic syndrome among patients with schizophrenia in Japan. Schizophr Res 123: 244-250.

Suvisaari, J., Saarni, S., Perala, J., Suvisaari, J., Harkanen, T., Lonnqvist, J. et al. (2007) Metabolic syndrome among persons with schizophrenia and other psychotic disorders in a general population survey. F Clin Psychiatry 68: 1045-1055.

Tandon, R., Nasrallah, H. and Keshavan, M. (2009) Schizophrenia, 'just the facts' 4 . Clinical features and conceptualization. Schizophr Res 110: 1-23.

Taylor, D. and McAskill, R. (2000) Atypical antipsychotics and weight gain - a systematic review. Acta Psychiatr Scand 101: 416-432.

Teixeira, P. and Rochal, F. (2007) The prevalence of metabolic syndrome among psychiatric inpatients in Brazil. Rev Bras Psiquiatr29: 330-336.

Teslovich, T., Musunuru, K., Smith, A., Edmondson, A., Stylianou, I., Koseki, M. et al. (2010) Biological, clinical and population relevance of 95 loci for blood lipids. Nature 466: 707-713.

Thakore, J., Mann, J., Vlahos, I., Martin, A. and Reznek, R. (2002) Increased visceral fat distribution in drug-naive and drug-free patients with schizophrenia. Int $\mathcal{F}$ Obes Relat Metab Disord 26: 137-141.

Tirupati, S. and Chua, L. (2007) Obesity and metabolic syndrome in a psychiatric rehabilitation service. Aust N Z F Psychiatry 41: 606-610.

Van Winkel, R., Rutten, B., Peerbooms, O., Peuskens, J., Van Os, J. and De Hert, M. (2010) MTHFR and risk of metabolic syndrome in patients with schizophrenia. Schizophr Res 121: 193-198.

Van Winkel, R., Van Os, J., Celic, I., Van Eyck, D., Wampers, M., Scheen, A. et al. (2008) Psychiatric diagnosis as an independent risk factor for metabolic disturbances: results from a comprehensive, naturalistic screening program. F Clin Psychiatry 69: 1319-1327.

Venkatasubramanian, G., Chittiprol, S., Neelakantachar, N., Naveen, M., Thirthall, J., Gangadhar, B. et al. (2007) Insulin and insulin-like growth factor-1 abnormalities in anti psychotic-naive schizophrenia. Am F Psychiatry 164: 1557-1560. 
Wang, J., Ban, M., Zou, G., Cao, H., Lin, T., Kennedy, B. et al. (2008) Polygenic determinants of severe hypertriglyceridemia. Hum Mol Genet 17: 2894.

Yazici, M., Anil Yagcioglu, A., Ertugrul, A., Eni, N., Karahan, S., Karaagaoglu, E. et al. (2011) The prevalence and clinical correlates of metabolic syndrome in patients with schizophrenia: findings from a cohort in Turkey. Eur Arch Psychiatry Clin Neurosci 261: 69-78.
Zabaneh, D. and Balding, D. (2010) A genome-wide association study of the metabolic syndrome in Indian Asian men. PloS One 5: e11961.

Zeggini, E., Scott, L., Saxena, R., Voight, B., Marchini, J., Hu, T. et al. (2008) Meta-analysis of genome-wide association data and large-scale replication identifies additional susceptibility loci for type 2 diabetes. Nat Genet 40: 638-645.
Visit SAGE journals online http://tpp.sagepub.com

(SAGE journals 